\title{
Local Wnt3a treatment restores bone regeneration in large osseous defects after surgical debridement of osteomyelitis
}

\author{
Johannes Maximilian Wagner ${ }^{1} \cdot$ Felix Reinkemeier $^{1} \cdot$ Mehran Dadras $^{1} \cdot$ Christoph Wallner $^{1} \cdot$ Julika Huber $^{1}$. \\ Alexander Sogorski ${ }^{1}$ - Maxi Sacher ${ }^{1}$. Sonja Schmidt ${ }^{1}$. Marius Drysch ${ }^{1}$. Stephanie Dittfeld ${ }^{1}$ • Mustafa Becerikli ${ }^{1}$. \\ Kathrin Becker ${ }^{2} \cdot$ Nicole Rauch $^{2} \cdot$ Marcus Lehnhardt $^{1} \cdot$ Björn Behr ${ }^{1}$ (D)
}

Received: 22 December 2019 / Revised: 13 April 2020 / Accepted: 11 May 2020 / Published online: 18 May 2020

(C) The Author(s) 2020, corrected publication 2021

\begin{abstract}
Impaired bone homeostasis caused by osteomyelitis provokes serious variations in the bone remodeling process, thereby involving multiple inflammatory cytokines to activate bone healing. We have previously established a mouse model for post-traumatic osteomyelitis and studied bone regeneration after sufficient debridement. Moreover, we could further characterize the postinfectious inflammatory state of bony defects after debridement with elevated osteoclasts and decreased bone formation despite the absence of bacteria. In this study, we investigated the positive effects of Wnt-pathway modulation on bone regeneration in our previous established mouse model. This was achieved by local application of Wnt3a, a recombinant activator of the canonical Wnt-pathway. Application of Wnt3a could enhance new bone formation, which was verified by histological and $\mu$-CT analysis. Moreover, histology and western blots revealed enhanced osteoblastogenesis and downregulated osteoclasts in a RANKL-dependent manner. Further analysis of Wnt-pathway showed downregulation after bone infections were reconstituted by application of Wnt3a. Interestingly, Wnt-inhibitory proteins Dickkopf 1 (DKK1), sclerostin, and secreted frizzled protein 1 (sFRP1) were upregulated simultaneously to Wnt-pathway activation, indicating a negative feedback for active form of Betacatenin. In this study, we could demonstrate enhanced bone formation in defects caused by post-traumatic osteomyelitis after Wnt3a application.
\end{abstract}

\section{Key messages}

- Osteomyelitis decreases bone regeneration

- Wnt3a restores bone healing after infection

- Canonical Wnt-pathway activation with negative feedback

Keywords Bone $\cdot$ Osteomyelitis $\cdot$ Wnt3a $\cdot$ Canonical Wnt-pathway $\cdot$ Bone regeneration

Johannes Maximilian Wagner and Felix Reinkemeier contributed equally to this work.

Electronic supplementary material The online version of this article (https://doi.org/10.1007/s00109-020-01924-9) contains supplementary material, which is available to authorized users.

\section{Björn Behr}

bjorn.behr@rub.de

1 University Hospital BG Bergmannsheil Bochum, Bürkle-de-la-Camp Platz 1, 44789 Bochum, Germany

2 Poliklinik für Kieferorthopädie, University Hospital Düsseldorf, Düsseldorf, Germany

\section{Background}

One of the main evolutionary driving forces in humans is the capability to combat infections. In this context, bone infections are of particular interest and need special attention as they pose one of the biggest problems in orthopedic surgery especially after open fractures of long bones [1]. The incidence of osteomyelitis (OM) in elective bone surgery is considered to be $1-5 \%$. However, OM still occurs in 5-30\% of open fractures, depending mainly on the severity of the fracture [1].

In the presence of necrotic bone material, the effectiveness of antibiotic therapy can be dramatically reduced due to biofilm formation [2]. The mainstay of treatment is therefore 
adequate surgical debridement, thus removing all purulent tissue.

In previous findings, we could demonstrate the essential status of surgical debridement versus antibiotic treatment alone in a murine tibia defect model [3], as eradication of bacteria could only be achieved by surgical intervention. Depending on the extent of infection, large bony defects can remain. Moreover, the bone in the vicinity still shows a dramatically reduced regenerative capacity despite complete absence of bacteria [3] after sufficient debridement. Accordingly, a further own study could identify an ongoing inflammatory reaction which leads to an upregulation of cytokines and activation of the innate immune system verified by elevated $B$ cells [4].

In a curative approach, the bone needs to be reconstructed or amputated. Immobility and mortality increases proportional to the level of amputation, given the higher energy and oxygen demand post-amputation [5]. For this reason, every effort should be attempted to limit the amount of bone loss by means of debridement and achieve bony reconstruction of the defects.

In bone biology, bone formation and resorption are highly complex regulated processes, which need to be balanced during physiological bone regeneration. Elevated bone resorption is a distinct feature of osteomyelitis, which leads to bone erosion quite similar to processes in rheumatoid arthritis [6]. In this context, plenty of mechanisms in bone inflammation have already been postulated, many involving the activation of the receptor activator nuclear factor- $\mathrm{KB}$ ligand (RANKL) which induces nuclear factor of activated $\mathrm{T}$ cells, cytoplasmic 1 (NFATc1), resulting in osteoclastogenesis [7]. Our recent work indicated elevated bone resorption after successful debridement of bone infections in mice [4]. This was mainly caused by an elevated osteoclastogenesis occurring together with an upregulation of RANKL activity. Interestingly, TNF- $\alpha$, which is known to be a main activator of RANKL pathway [8], was not involved in this inflammatory process. Regarding increased bone resorption, distal-less homebox protein 5 (DLX-5) is known to be crucial for osteoblastosteoclast interaction and osteoclast regulation [9] and decreased levels of DLX-5 are associated to an increased bone resorption.

Wnt-pathway is an important pathway for plenty of different regenerative processes.

In canonical Wnt-pathway, Beta-catenin is formed continuously. While the Wnt-pathway is inactivated, a complex consisting of axis inhibition protein (Axin), adenomatous polyposis coli (APC), glycogen synthase kinase 3 beta (GSK-3 $\beta$ ), and casein kinase 1 alpha $(\mathrm{CK} 1 \alpha)$ phosphorylates and thereby inactivates Beta-catenin [10, 11]. Pathway activation leads to an increased recruitment of Disheveled (Dvl), which in turn inhibits the inactivation complex [12]. Activated Beta-catenin translocates into nucleus and acts as a co-activator of transcription factor (T Cell Specific, HMGBox) (TCF)/lymphoid enhancer-binding factor (LEF) [13].

It has already been shown that Wnt3a is a strong promoter of bone formation in differentiated cells [14-20]. Moreover, Wnt-pathway is known to increase angiogenesis in various tissues and organs, mainly via increased expression of vascular endothelial growth factor (VEGF) [21]. However, studies investigating Wnt-pathway in the setting of inflammatory diseases are sparse.

In the light of altered bone regeneration after post-traumatic osteomyelitis, we sought to improve bone healing through direct activation of canonical Wnt-pathway.

Thus, we applied Wnt3a locally after debridement in our established murine bone infection model [3]. Decreased bone regeneration after infection could be restored via increased osteogenesis and inhibition of osteoclast activity.

\section{Methods}

\section{Mouse osteomyelitis model}

Animals were housed and caged individually with free access to water and food under specific pathogen-free conditions. C57BL/6 J male and female mice, 12 weeks old with an average weight of $25 \mathrm{~g}$, were used for this project. Surgical steps were performed, as previously described [3] in our previously established animal model. Briefly, a skin incision was placed over the proximal medial tibia, and a hole (1 $\mathrm{mm}$ in diameter) was drilled into the proximal medial tibia after retraction of the tibialis anterior muscle. Thereafter, S. aureus in a concentration of 1000 units per $\mu$ l was injected into the medullary cavity of the tibia. Infection of the tibia was performed in all experimental animals in this study except for animals in a group of uninfected control. Thereafter, the muscle was reapproximated and the wound closed.

Two weeks after Staph. aureus inoculation, a second surgery for sufficient debridement was performed. The skin incision was placed once again over the proximal medial tibia exposing the bone defect. Then, infected lytic bone tissue was debrided and completely removed, followed by rinsing of debrided defects with isotone sodium chloride solution.

Thereafter, 1- $\mu \mathrm{g}$ Wnt3a (recombinant mouse Wnt3a, Peprotech, Rocky Hill, USA) in $1 \mathrm{ml}$ was placed into the bony defect on a collagen sponge. As a control, $1 \mathrm{ml}$ of PBS was administered to the bone defect on a collagen sponge as well. Moreover, infected animals (OM) and uninfected control (control) received no treatment.

Mice were sacrificed 3 and 7 days after debridement which represents an early and late time point during bone regeneration, according to the experiences of Colnot et al. [22]. 


\section{Protein isolation}

For protein isolation, only the bony defect of 9 animals per group was used, removing all of the remaining parts of the bone except for the region of interest.

Thereafter, the bony defect was snap frozen after harvest and stored at $-80^{\circ} \mathrm{C}$. For further processing of the specimens, bony defects were frozen in liquid nitrogen and pestled into smaller bone fragments. Then, bone fragments were collected and homogenized in lysis buffer, containing protease inhibitors (aprotinin, leupeptin, pepstatin A, and phosphatase inhibitor), until lysis was completed. Then, cellular debris was removed by centrifugation, and isolated protein was stored at $80^{\circ} \mathrm{C}$ for further experiments.

\section{Western blot}

Isolated protein was combined and mixed with Laemmli sample buffer. After denaturation at $95{ }^{\circ} \mathrm{C}$, samples were directly loaded onto the SDS PAGE. Fifteen percent polyacrylamide gels were used for electrophoresis of 30- $\mu$ g total protein per lane. Protein was transferred to a nitrocellulose membrane using wet transfer method before membranes were blocked with $3 \%$ bovine serum albumin to prevent unspecific binding. After blocking, membranes were washed again in TBS-T to remove remaining blocking solution. Hereafter, membranes were incubated with primary antibodies against Beta-catenin, GSK-3 $\beta$ (cat. nr. 19807S, 5558S) (cell signaling technology, Danvers, USA), RANKL, Runx2, DKK1, sclerostin, sFRP1, and osteoprotegerin (cat.nr. ab45039, ab23981, ab61275, ab63097, ab4193, ab183910) (Abcam, Cambridge, UK) overnight at $4{ }^{\circ} \mathrm{C}$, followed by washing and incubation with HRPconjugated secondary antibody (Thermo Fisher Scientific, Waltham, USA; Santa Cruz Biotechnologies, Dallas, USA). Both antibodies were diluted in BSA. Proteins were detected for 30-60 s by enhanced chemoluminescence using Kodak Image Station 4000 mm (Kodak, Rochester, USA) according to standard protocol [23].

\section{Micro-computed tomographic analysis}

Bone specimens of 3 animals per group were scanned with a $\mu C T$ device (Viva CT 80; Scanco Medical AG, Brüttisellen, Switzerland) operated at $70 \mathrm{kVp}, 114 \mu \mathrm{A}, 8 \mathrm{~W}, 31.9-\mathrm{mm}$ $\mathrm{FOV}$, an integration time of $1167 \mathrm{~ms}$, and $2 \mathrm{x}$ frame averaging. The data sets were reconstructed into $3 \mathrm{D}$ volumes with an isotropic nominal resolution of $15.6-\mu \mathrm{m}$ voxel size.

\section{Image processing}

Further processing of the scanned images was performed using $\mu$-CT Evaluation Software Program V6.5 (Scanco Medical AG, Brüttisellen, Switzerland). A standardized cylindrical volume of interest with $16.84 \mathrm{~mm}$ in diameter was placed within the defect site, defining the region of interest. Thereafter bone volume to total volume (BV/TV) was assessed according to the guidelines for assessment of bone microstructures using $\mu \mathrm{CT}$ (Bouxsein, Guidelines for assessment of bone microstructure in rodents using micro-computed tomography 2010).

\section{Histology, immunohistochemistry, and immunofluorescence}

For all histological procedures, tibiae of 6 mice per group were taken and fixed in $4 \%$ paraformaldehyde solution overnight, decalcified in 19\% EDTA solution, and finally paraffin embedded. Thereafter, tibiae were longitudinally sectioned at $9 \mu \mathrm{m}$. Following, aniline blue staining was performed as previously described [24]. Images were taken with Zeiss Axivert 100 (Zeiss, Oberkochen, Germany). Quantification of aniline blue positive pixels was performed in Adobe Photoshop placing a $2000 \times 2000$ pixel dimension selection box over the entire defect area. The magic wand tool (tolerance $60 \%$; non-contiguous) was used to mark aniline blue stained osteoid formation semi-automatically. Thereafter, cortical bone was deselected manually. The highlighted pixels reliably corresponded to the new bone formation area.

Furthermore, TRAP-staining was performed with TRAP Kit (Sigma-Aldrich, St. Louis, USA) according to the manufacturers' instruction.

Additionally, immunohistochemical staining were performed using Vectastain ABC Kit (Vector Laboratories, Burlingame, USA) with primary antibodies against osteocalcin, PCNA (cat. nr. sc-30,045, sc-7907) (Santa Cruz Biotechnologies, Dallas, USA) and CD31 (cat. nr. 553,700) (BD Biosciences, Franklin Lakes, USA). After deparaffinization and rehydration of bone sections after standard protocols, antigen demasking was performed using $1 \%$ proteinase $\mathrm{K}$ solution. Thereafter, specimens were incubated with $3 \%$ hydrogen peroxide solution to quench endogenous peroxidase activity. Thereafter, specimens were blocked with normal blocking serum to prevent unspecific binding of primary antibody that was subsequently applicated and incubated overnight at $4{ }^{\circ} \mathrm{C}$. Following, secondary antibody conjugated to horseradish peroxidase (HRP) was used, and staining reaction was performed by the use of NovaRED (HRP) Peroxidase Substrate Kit (Vector Laboratories, Burlingame, USA).

For immunofluorescent staining, primary antibodies against Runx2 (cat. nr. sc-10,758) (Santa Cruz Biotechnologies, Dallas, USA), Beta-catenin, GSK-3 $\beta$ (cat. nr. 19807S, 5558S) (cell signaling technology, 
Danvers, USA) were used. Initial steps were carried out similar to immunohistochemical staining until application of primary antibody. Thereafter, samples were incubated with secondary antibody conjugated to Alexa Fluor594 (Thermo Fisher Scientific, Waltham, USA). Images were taken with Zeiss Axioplan microscope. Quantification of immunohistochemistry was performed selecting a region of interest of $2000 \times 2000 \mathrm{Px}$ and automatic selection via magic wand tool (tolerance $60 \%$; noncontiguous). Quantification of TRAP stain pixels was carried out in relation to pixels of bone surface according to standard protocol [25].

\section{Statistics}

Results are presented as mean \pm standard error of the mean (SEM) of at least three independent experiments. ShapiroWilk test was used to check for normal distribution. Additionally, f-test was used to examine variances of both groups.

$p$ values were calculated by student's $t$ test comparing two groups. For post hoc comparisons, Tukey's test was used. Statistical significances were set at a $p$ value $<0.05$.

\section{Results}

\section{Canonical Wnt-pathway is downregulated in post- infectious inflammatory state}

In order to check canonical Wnt-pathway activity, immunoflourescent staining against glycogen synthase kinase (GSK-3 $\beta$ ) and Beta-catenin were performed. Infected animals, which underwent no further treatment showed enhanced signaling of GSK-3 $\beta$ and decreased Beta-catenin in comparison with the uninfected control, indicating decreased activity of the canonical Wntpathway due to infection (Fig. 1B).

\section{Wnt3a application enhances osteogenesis and angiogenesis after osteomyelitis}

After application of Wnt3a, our first interest was to evaluate new bone formation upon treatment. Aniline blue staining (Fig. 2A) and $\mu-\mathrm{CT}$ analysis (Fig. 2C and D) revealed significantly increased osteogenesis 7 days after Wnt3a application. Accordingly, elevated osteoblastogenesis and mature osteoblasts could be observed in runtrelated transcription factor 2 (Runx2) and osteocalcin staining (Fig. 2B), as well as western blots (Fig. 3). Moreover, distal-less homebox protein 5 (DLX-5), which is known to be a potent modulator of Runx 2 and thereby osteoblast differentiation, was also upregulated by Wnt3a a

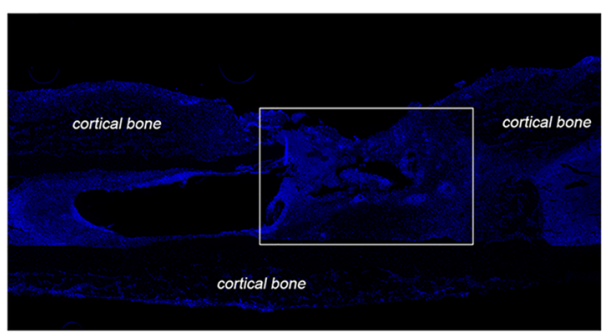

b

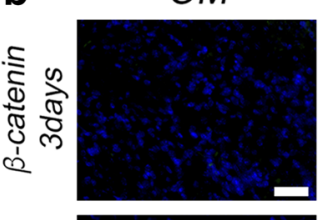

$O M$

\section{Control}
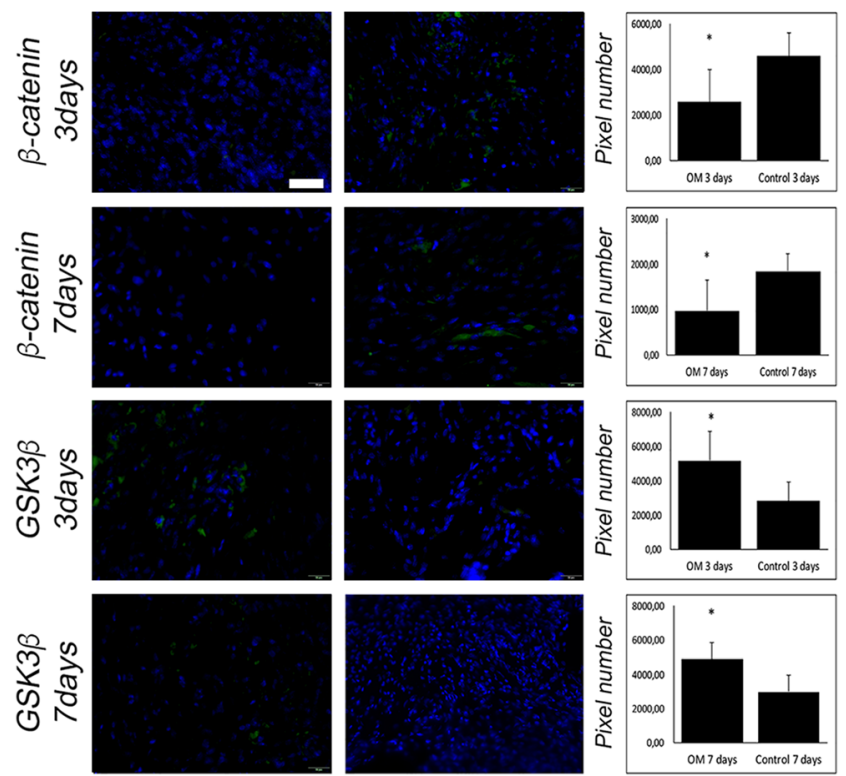

Fig. 1 Postinfectious inflammatory state of osteomyelitis impairs canonical Wnt-pathway. (A) Overview of immunofluorescent images of the tibial bone, DAPI-stain (blue). The white square marks the region of interest of all immunoflourescent and immunohistochemical staining. (B) Immunoflourescent staining against $\beta$-catenin (green), GSK-3 $\beta$ (green) and DAPI (blue) of infected and debrided animals (OM) and uninfected control animals (Control). Y-axis stands for pixel number of stained pixel quantification. Scale bar represents $20 \mu \mathrm{m} ; p$ value, $*<0.05$

application (Fig. 3). Furthermore, staining detecting Proliferating-Cell-Nuclear-Antigen (PCNA) revealed enhanced proliferation after Wnt3a treatment (Fig. 4A). Interestingly, staining against PECAM-1 showed increased angiogenesis after activation of canonical Wntpathway (Fig. 4A).

\section{Osteoclast activity can be decreased by canonical Wnt-pathway activation}

Elevated osteoclast activity was one of the main reasons for altered bone regeneration during inflammatory state after post-traumatic osteomyelitis. As Wnt-pathway activation is known to diminish osteoclast activity ${ }^{20}$, we were interested to investigate osteoclasts after activation of canonical Wntpathway. 
a

\section{Wnt3a 7 days quantified pixels}

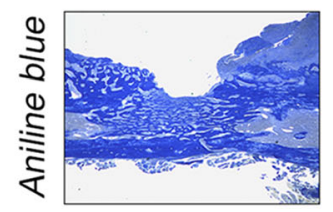

b Wnt3a 3 days
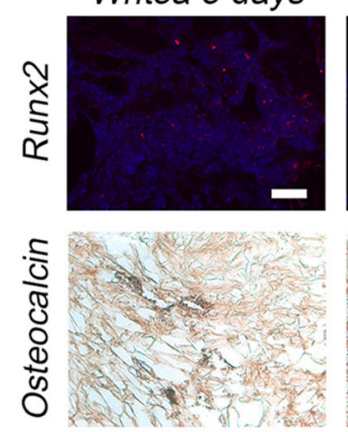

C
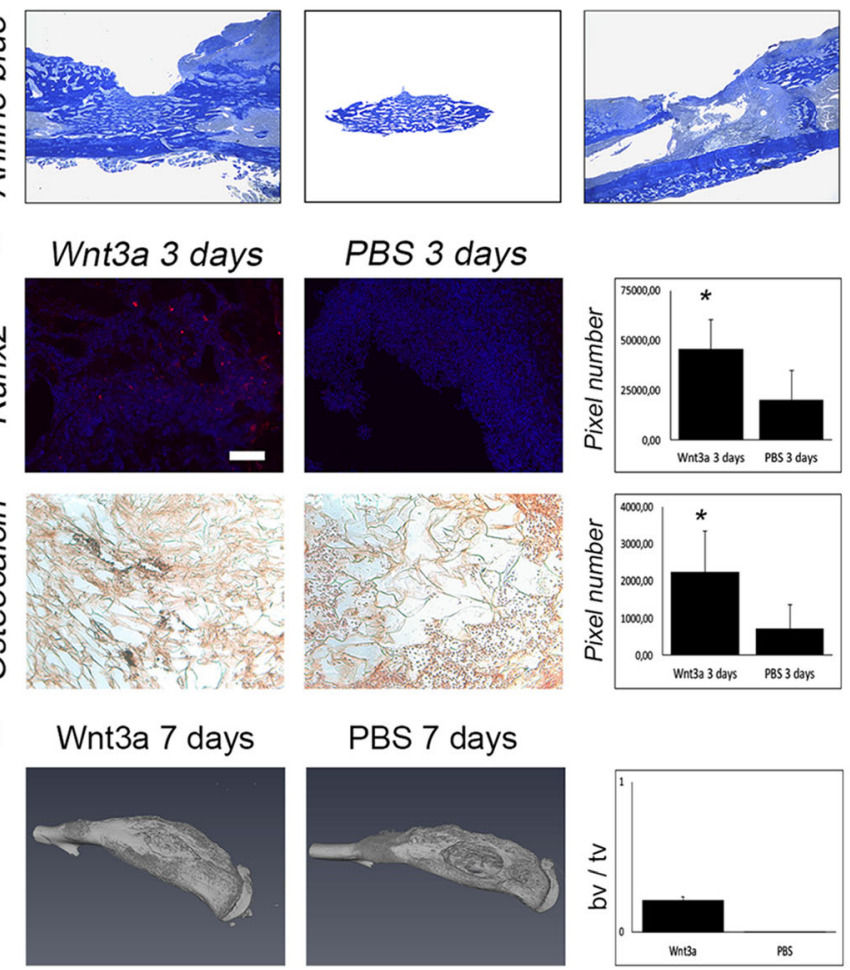

Fig. 2 Enhanced osteogenesis and osteoblastogenesis due to Wnt3a treatment. Overview of tibial defect and region of interest (red square) of immunofluorescent and immunohistochemical staining (above). (A) Aniline blue staining and (B) immunoflourescent staining against Runx2 (red) and DAPI (blue) and immunohistochemical staining against osteocalcin (red) of infected animals treated by Wnt3a or PBS. Y-axis stands for pixel number of stained pixel quantification. Scale bar aniline

Subsequently, after Wnt3a application, animals showed less osteoclast activity in TRAP-staining (Fig. 4A) accompanied by decreased levels of receptor quantified pixels
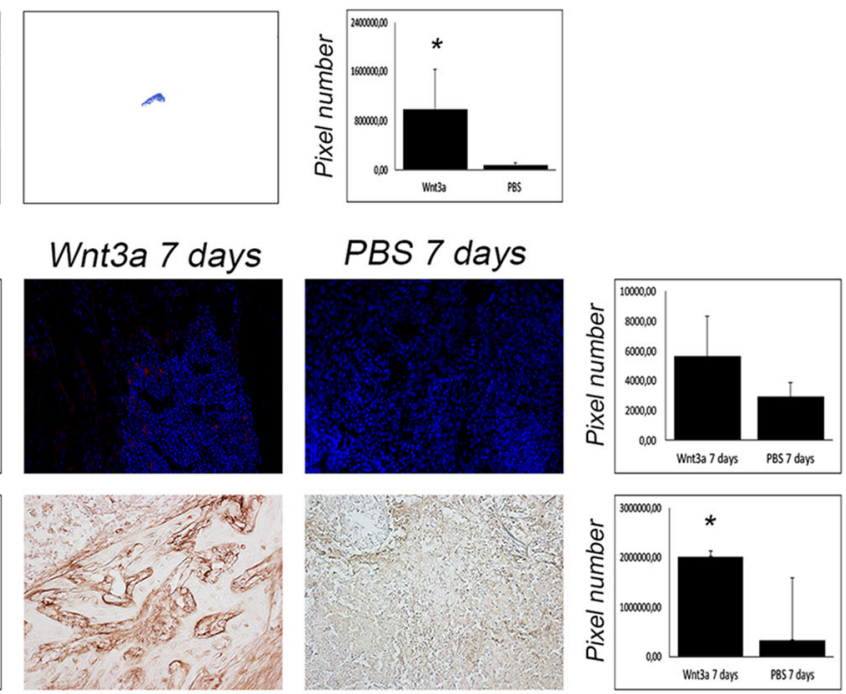

d

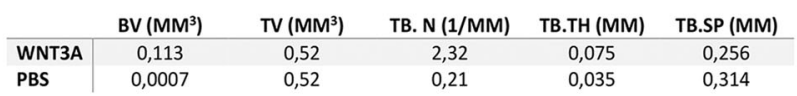

blue represents $400 \mu \mathrm{m}$; Scale bar Runx 2 and osteocalcin represents $100 \mu \mathrm{m} ; p$ value, $*<0.05$. (C) $\mu \mathrm{CT}$ scans of Wnt3a and PBS group tibia. $\mathrm{Y}$-axis stands for bone volume to total volume (bv/tv) measured within defect site. (D) bone volume (BV) in $\mathrm{mm}^{3}$, total volume (TV) in $\mathrm{mm}^{3}$, trabecular number (TB.N) in $1 / \mathrm{mm}$, trabecular thickness (TB.TH) in mm, and trabecular separation (TB.SP) in $\mathrm{mm}$ of region of interest of $\mu \mathrm{CT}$ scans of WNT3a and PBS group

activator of NF-KB Ligand (RANKL) and elevated levels of osteoprotegerin in western blot (Fig. 4B). 


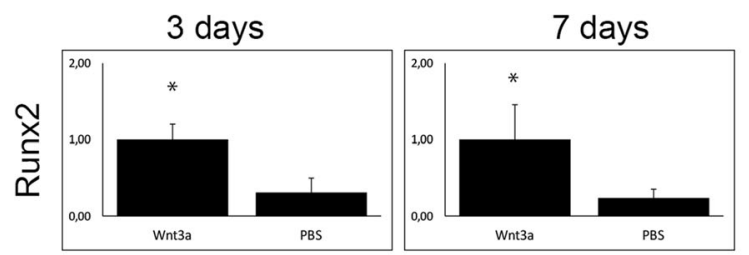

3 days
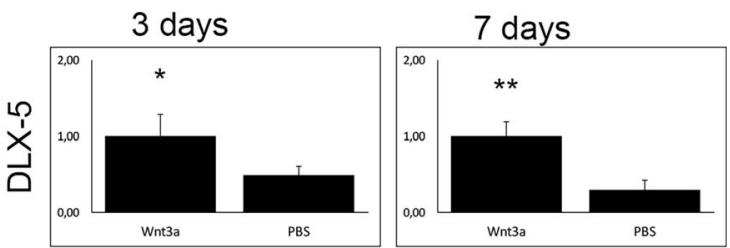

7 days

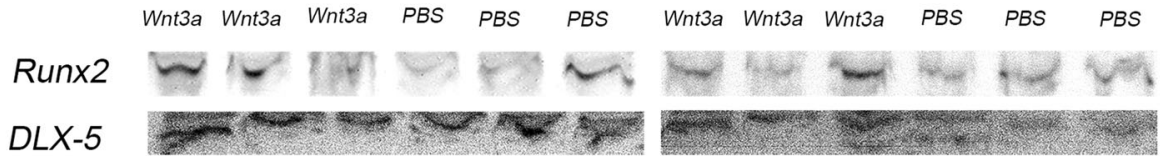

Fig. 3 Western blots verified upregulated osteoblastogenesis due to Wnt3a activation. Data obtained from western blots of WNT3a and PBS group after 3 and 7 days and cropped images of western blots

\section{Wnt-pathway inhibitors are upregulated upon Wnt3a application}

Addition of Wnt3a subsequently restored canonical Wntpathway activity, whereas decreased Wnt-activity could be seen in PBS-treated control animals, similar to infected animals which received no further treatment (Fig. 5A). against markers for osteoblastogenesis (Runx2, DLX5). $p$ value, $*<$ $0.05, * *<0.01$

In accordance, western blot showed similar findings of GSK-3 $\beta$ and Beta-catenin levels (Fig. 5B).

Besides Wnt-pathway activity, our interest was to elucidate the orchestration of activation and inhibition of the Wnt-pathway. Interestingly, inhibitory proteins, Dickkopf-related protein (DKK1), secreted frizzled-related protein 1 (sFRP1), and sclerostin all seemed to be upregulated in the Wnt3a group
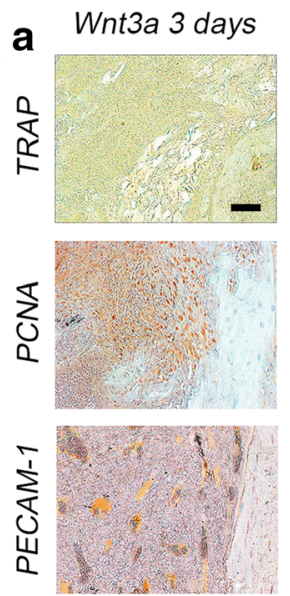

b
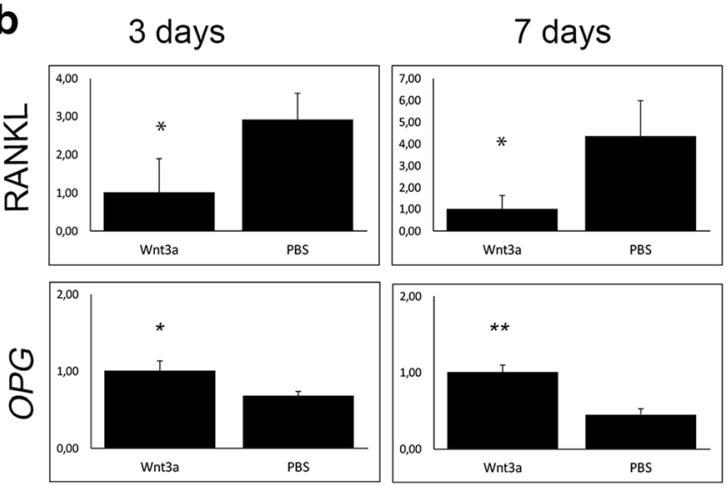

Fig. 4 Wnt-pathway activation leads to diminished osteoclasts and increased angiogenesis and proliferation. (A) TRAP staining and immunohistochemical staining against PCNA (red) and PECAM-1 (red) of infected Wnt3a and PBS treated animals 3 and 7 days after treatment. Y-Axis stands for pixel number of stained pixel quantification.
Wnt3a 7 days
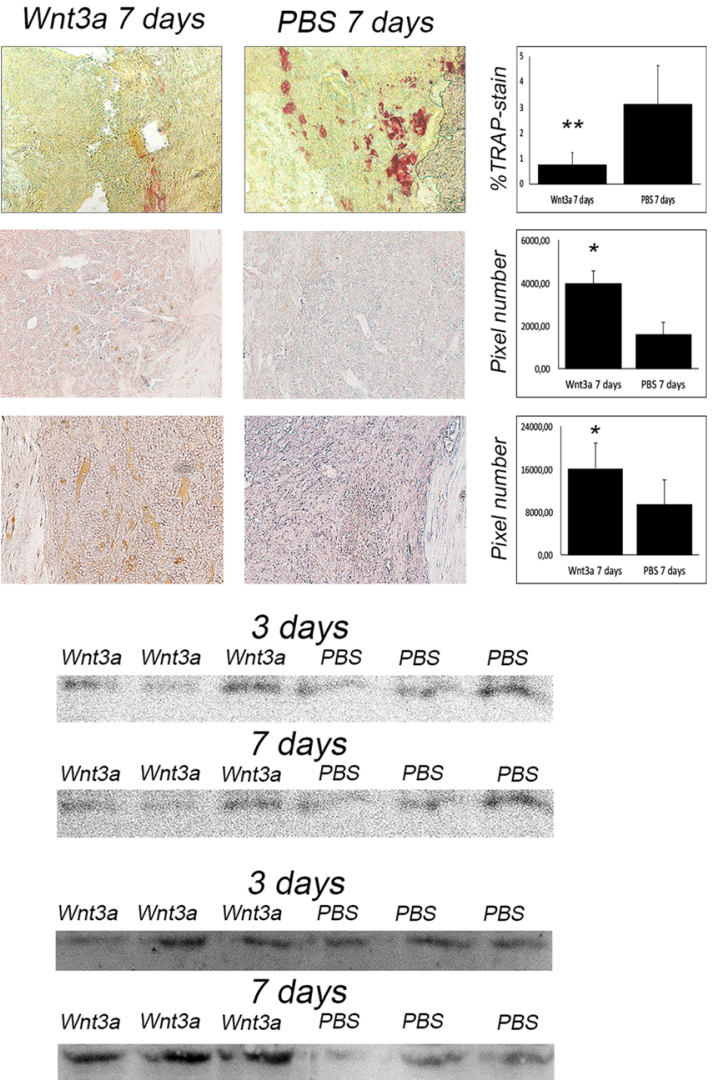

Scale bar represents $100 \mu \mathrm{m} ; p$ value, ${ }^{*}<0.05$. (B) Western blot data and cropped images of Wnt3a and PBS group after 3 and 7 days of markers concerning osteoclastogenesis (RANKL, OPG). $p$ value, $*<0.05, * *<$ 0.01 


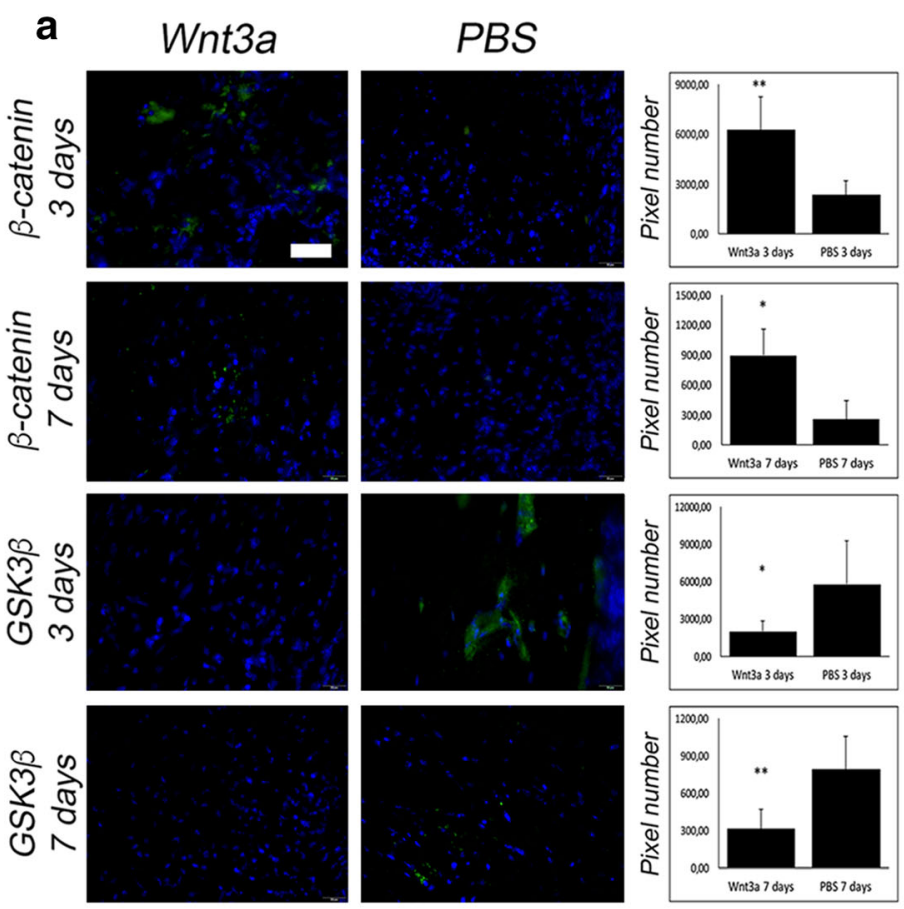

b
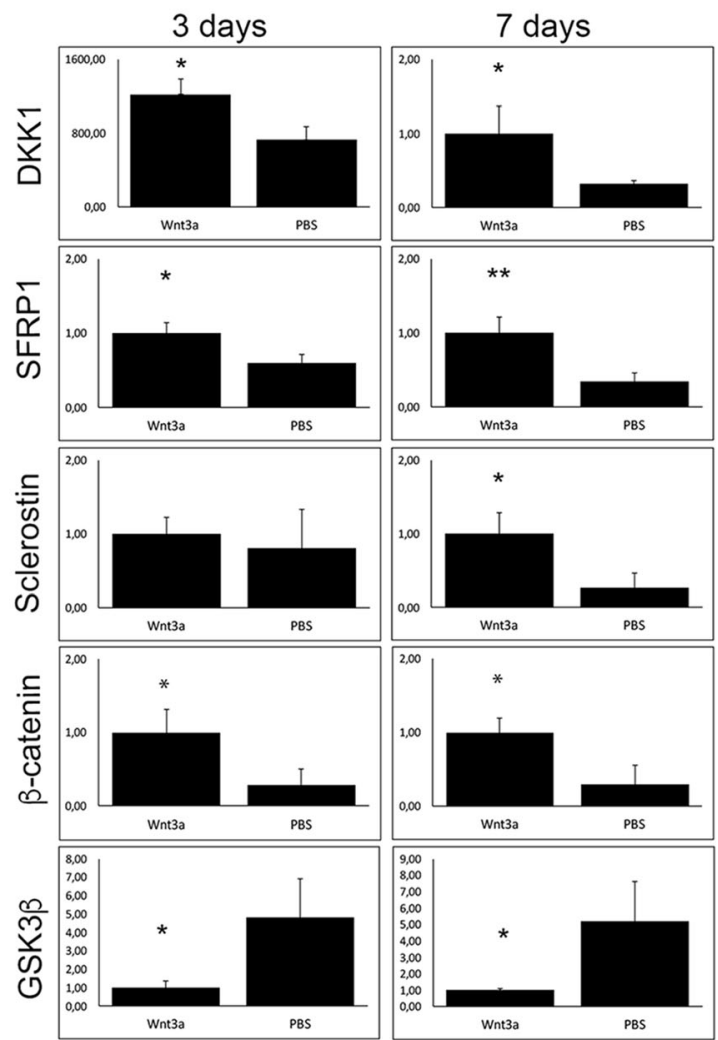

3 days

7 days Wnt3a Wnt3a Wnt3a PBS PBS PBS Wnt3a Wnt3a Wnt3a PBS PBS PBS

$\beta$-catenin

GSK3 $\beta$

$D K K 1$

SFRP1

Sclerostin
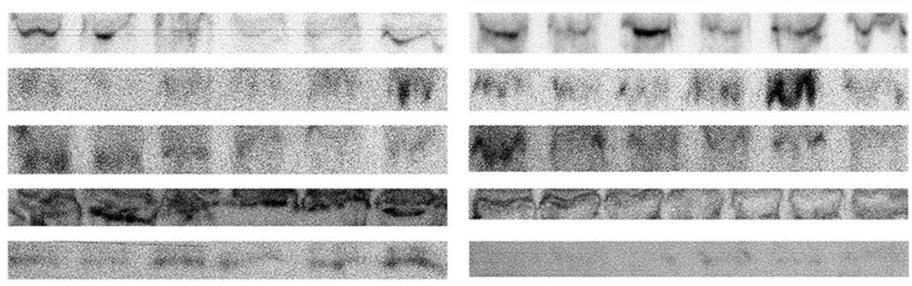

Fig. 5 Wnt3a application activates Wnt-pathway. (A) Immunoflourescent staining against Beta-catenin (green) and GSK-3 $\beta$ (green) and DAPI (blue) of infected and Wnt3a and PBS treated animals. Y-axis stands for pixel number of stained pixel quantification. Scale bar

(Fig. 5B). Except sclerostin, which was only regulated on day 7 , all of these factors were elevated on both day 3 and 7 .

\section{Discussion}

It is well accepted that inflammatory processes diminish regenerative healing capacity. Especially in bone infections, this is a common problem of orthopedic surgery [26]. Based on previous studies [3, 4], this work aimed to improve bone healing via activation of canonical Wnt-pathway and reveal Wnt-dependent mechanisms of bone formation and resorption related to bone infection. represents $20 \mu \mathrm{m} ; p$ value, $*<0.05, * *<0.01$. (B) Western blot data and cropped images of Wnt $3 \mathrm{a}$ and PBS group after 3 and 7 days of Wntpathway activity markers (Beta-catenin, GSK-3 $\beta$, DKK1, SFRP1, sclerostin). $p$ value, $*<0.05, * *<0.01$

Our focus was to evaluate the regenerative capacity of Wnt3a-treated mice in a murine post-traumatic osteomyelitis model. Histology and western blots revealed elevated bone formation, osteoblastogenesis, and angiogenesis. Interestingly, these differences could be seen throughout the observation period, during early and late bone regeneration, despite a single Wnt3a treatment at the beginning.

Osteogenic markers Runx2 and DLX-5 were significantly upregulated in Wnt3a group, suggesting a regulation via Wntpathway. In this context, direct activation of Runx 2 by Betacatenin has already been demonstrated in various settings [27-29] and proved to be an effective treatment strategy to enhance bone regeneration in long bone fractures [27]. 
DLX-5 is known to be crucial for endochondral ossification and moreover regulates osteogenic factors like Runx 2 and Osterix [30-32]. Typically activated by the bone morphogenetic protein (BMP) pathway [30,33], only little is known about Wnt pathway interaction. Elevated BMP-2 and thereby DLX-5 levels have been described after canonical Wntpathway activation [34].

Similarly to our observations, activation of canonical Wntpathway is known to be a strong promoter of angiogenesis during physiological and pathological conditions, regulating VEGF and other factors crucial for angiogenesis [21].

Concerning bone resorption, we could observe a markedly diminished osteoclasts activity presumably regulated by the RANKL/OPG axis via activation of canonical Wnt-pathway. Especially patients with inflammatory joint diseases like rheumatoid arthritis suffer from excessive RANKL expression and enhanced bone resorption [35], making it therefore a therapeutical target in these patients [36]. Moreover, a deregulation of the RANKL/OPG axis is consistent to our previous work, characterizing bone healing after osteomyelitis, revealing a post-infectious inflammatory state with elevated osteoclast activity and diminished osteoblastogenesis [4].

It is well accepted that Wnt3a and other activators of canonical Wnt-pathway are strong promoters of bone regeneration [14-20], enhancing osteoblastogenesis and thereby bone formation. Besides that, bone resorption is known to be affected by canonical Wnt-pathway, decreasing osteoclast activity via modulation of osteoclast progenitor cell differentiation and RANKL/OPG axis [37-39].

To verify canonical Wnt-pathway activation, the active form of Beta-catenin and GSK-3 $\beta$ was investigated. In Wnt3a group, Wnt-pathway activation was markedly increased throughout the observation period. However, only little is known about Wnt-pathway activation in the setting of inflammatory response after bone infection.

It has been shown that osteoblasts in the vicinity of inflamed joints in rheumatoid arthritis (RA) showed less osteogenic markers while upregulation of Wnt inhibitors DKK1 and Frizzled-related proteins [40].

Moreover, blocking antibodies of DKK-1 promotes bone formation and prevents bone erosion [41]. Interestingly, activated canonical Wnt-signaling might also have immunomodulatory roles as activated Beta-catenin can reduce bacteriainduced inflammation [42]. In a Salmonella infection model, constitutive activation of Beta-catenin (and thereby canonical Wnt-signaling) showed inhibition of NF-kB-activity [43].

Wnt-pathway is a highly complex-regulated network involving different agonists and inhibitory factors. Some of the most important inhibitory factors, namely DKK1, sFRP1, and sclerostin, were all elevated after Wnt3a treatment.

DKK1 is a direct inhibitor of canonical Wnt-pathway. Interestingly, a negative feedback loop, increasing the expression of DKK1 initiated via Beta-catenin is well known [44-46]. Moreover, activation of Wnt/Beta-catenin pathway by neutralizing antibodies against sclerostin was accompanied by increased levels of sclerostin and DKK1 $[47,48]$ This leads to the assumption of a negative-feedback between canonical Wnt-pathway and sclerostin as well and thus limiting Wntdriven bone formation. Consistently, a dual inhibition of sclerostin and DKK1 leads to increased bone formation in comparison with inhibition of each one of them separately [48]. For example, in patients with type 2 diabetic osteoporosis, a close link of bone loss to the canonical Wnt pathway with increased expression of DKK1 and sclerostin became evident in a recently published study [49]. Within this context, upregulated levels of sFRP1 are also associated with different metabolic bone disorders including osteoporosis, mainly because of Wnt/Beta-catenin inhibition [50]. Originally, being identified as a Wnt inhibitor, more recent studies revealed a more differentiated role of sFRP1 in Wnt signaling. Depending on the cellular context, concentration and expression patterns of Fzd receptors sFRP1 could either promote or inhibit Wnt/Beta-catenin pathway. Interestingly, a direct link between Hedgehog signaling pathway and Beta-catenin was described. Downregulation of the Hedgehog signaling via glioma-associated oncogene homolog 3 (GLI3) leads in turn to a downregulation of sFRP1 [51]. Accordingly, the upregulation of sFRP1 after Wnt3a application in this work seems striking. However, we propose another regulatory mechanism leading to upregulation of SFRP1 in this case, as the regulation of sFRPs in Wnt pathway is not fully understood and needs to be further investigated. In fact, $\mathrm{sFRP} 1$ has protective regulatory Wnt-related effects against carcinomas in various cell types, making this assumption more likely [50].

The anabolic functions of Wnt/Beta-catenin pathway could restore the bone healing deficiencies after post-traumatic osteomyelitis in long bone. Local Wnt3a application led to increased bone formation, affecting both osteoblasts via Runx2 and DLX-5 upregulation and osteoclasts shifting RANKL/ OPG-axis. Moreover, the osteogenic capacity of canonical Wnt-pathway activation was regulated by elevated Wnt inhibitors DKK1 and sclerostin.

Activating Wnt-pathway could promote bone regeneration of patients with large bony defects after radical debridement of osteomyelitis. To date, commercially available antibodies against sclerostin exist, being used to increase bone mass in postmenopausal osteopenia [52].

\section{Conclusions}

The findings of this study could show the beneficial effects of Wnt3a application on bone regeneration after post-traumatic osteomyelitis. Subsequently, activation of canonical Wntpathway leads to increased bone formation and 
osteoblastogenesis, whereas osteoclast activity could be decreased. Interestingly, activation of Wnt/Beta-catenin pathway elevated pathway inhibitors DKK1, sclerostin, and sFRP1. Thus, a negative feedback of active Beta-catenin could be assumed.

Authors' contributions JW, FR, SD, SS, and MD did all the experiments and BB, JW, FR, MD, CW, JH, SS, SD, HJ, MB, and ML interpreted data and contributed to research design. All authors have read and accepted the final version of the manuscript.

Funding information Open Access funding enabled and organized by Projekt DEAL. This work was supported by a grant of the DFG (Deutsche Forschungsgemeinschaft) (BE 4169/8-1).

\section{Compliance with ethical standards}

Conflict of interest The authors declare that they have no conflicts of interests.

Ethics approval All experiments were performed in adherence to the National Institute of Health guidelines for the use of experimental animals and after approval by the German legislation.

The protocol was approved by the LANUV (NRW, Germany; PermitNumber: 84-02.04.2014.A044).

Abbreviations OM, osteomyelitis; RANKL, receptor activator nuclear factor- $\mathrm{KB}$ ligand; OPG, osteoprotegerin; NFATc1, nuclear factor of activated T cells, cytoplasmic 1 ; TNF- $\alpha$, tumor necrosis factor alpha; Axin, axis inhibition protein; APC, adenomatous polyposis coli; GSK- $3 \beta$, glycogen synthase kinase 3 beta; $\mathrm{CK} 1 \alpha$, casein kinase 1 alpha; Dvl, Disheveled; Runx 2, runt-related transcription factor 2; DKK1, Dickkopfrelated protein 1; PCNA, Proliferating-Cell-Nuclear-Antigen; sFRP1, secreted frizzled-related protein 1; DLX-5, distal-less homebox protein 5; PECAM-1, platelet endothelial cell adhesion molecule 1; LRP , lowdensity lipoprotein receptor related protein

Open Access This article is licensed under a Creative Commons Attribution 4.0 International License, which permits use, sharing, adaptation, distribution and reproduction in any medium or format, as long as you give appropriate credit to the original author(s) and the source, provide a link to the Creative Commons licence, and indicate if changes were made. The images or other third party material in this article are included in the article's Creative Commons licence, unless indicated otherwise in a credit line to the material. If material is not included in the article's Creative Commons licence and your intended use is not permitted by statutory regulation or exceeds the permitted use, you will need to obtain permission directly from the copyright holder. To view a copy of this licence, visit http://creativecommons.org/licenses/by/4.0/.

\section{References}

1. Lew DP, Waldvogel FA (2004) Osteomyelitis. Lancet 364:369379

2. Costerton JW (2006) Biofilm theory can guide the treatment of device-related orthopaedic infections. Clin Orthop Relat Res 437: $7-11$

3. Wagner JM, Zollner H, Wallner C, Ismer B, Schira J, Abraham S, Harati K, Lehnhardt M, Behr B (2016) Surgical debridement is superior to sole antibiotic therapy in a novel murine posttraumatic osteomyelitis model. PLoS One 11:e149389
4. Wagner JM, Jaurich $\mathrm{H}$, Wallner C, Abraham S, Becerikli M, Dadras M, Harati K, Duhan V, Khairnar V, Lehnhardt M, Behr B (2017) Diminished bone regeneration after debridement of posttraumatic osteomyelitis is accompanied by altered cytokine levels, elevated B cell activity and increased osteoclast activity. J Orthop Res 35:2425-2434

5. Pinzur MS, Gold J, Schwartz D, Gross N (1992) Energy demands for walking in dysvascular amputees as related to the level of amputation. Orthopedics 15:1033-1037

6. Jones D, Glimcher LH, Aliprantis AO (2011) Osteoimmunology at the nexus of arthritis, osteoporosis, cancer, and infection. J Clin Invest 121:2534-2542

7. Takayanagi H (2012) New developments in osteoimmunology. Nat Rev Rheumatol 8:684-689

8. Wei S, Kitaura H, Zhou P, Ross FP, Teitelbaum SL (2005) IL-1 mediates TNF-induced osteoclastogenesis. J Clin Invest 115:282290

9. Samee N, Geoffroy V, Marty C, Schiltz C, Vieux-Rochas M, Levi G, de Vernejoul M-C (2008) Dlx5, a positive regulator of osteoblastogenesis, is essential for osteoblast-osteoclast coupling. Am J Pathol 173:773-780

10. Xue B, Dunker AK, Uversky VN (2012) The roles of intrinsic disorder in orchestrating the Wnt-pathway. J Biomol Struct Dyn 29:843-861

11. Seo E, E-h J (2007) Axin-independent phosphorylation of APC controls beta-catenin signaling via cytoplasmic retention of betacatenin. Biochem Biophys Res Commun 357:81-86

12. Clevers $\mathrm{H}$ (2006) $\mathrm{Wnt} /$ beta-catenin signaling in development and disease. Cell 127:469-480

13. Behrens J, von Kries JP, Kuhl M, Bruhn L, Wedlich D, Grosschedl R, Birchmeier W (1996) Functional interaction of beta-catenin with the transcription factor LEF-1. Nature 382:638-642

14. Quarto N, Behr B, Longaker MT (2010) Opposite spectrum of activity of canonical Wnt signaling in the osteogenic context of undifferentiated and differentiated mesenchymal cells: implications for tissue engineering. Tissue Eng Part A 16:3185-3197

15. Minear S, Leucht P, Jiang J, Liu B, Zeng A, Fuerer C, Nusse R, Helms JA (2010) Wnt proteins promote bone regeneration. Sci Transl Med 2:29ra30

16. Arioka M, Takahashi-Yanaga F, Sasaki M, Yoshihara T, Morimoto S, Takashima A, Mori Y, Sasaguri T (2013) Acceleration of bone development and regeneration through the Wnt/beta-catenin signaling pathway in mice heterozygously deficient for GSK-3beta. Biochem Biophys Res Commun 440:677-682

17. Dixit M, Raghuvanshi A, Gupta CP, Kureel J, Mansoori MN, Shukla P, John AA, Singh K, Purohit D, Awasthi P, Singh D, Goel A (2015) Medicarpin, a natural pterocarpan, heals cortical bone defect by activation of notch and Wnt canonical signaling pathways. PLoS One 10:e0144541

18. Wei Q, Zhang J, Hong G, Chen Z, Deng W, He W, Chen MH (2016) Icariin promotes osteogenic differentiation of rat bone marrow stromal cells by activating the ERalpha-Wnt/beta-catenin signaling pathway. Biomed Pharmacother 84:931-939

19. Zhang X, Chen Q, Liu J, Fan C, Wei Q, Chen Z, Mao X (2017) Parthenolide promotes differentiation of osteoblasts through the $\mathrm{Wnt} /$ beta-catenin signaling pathway in inflammatory environments. J Interferon Cytokine Res 37:406-414

20. Leucht P, Lee S, Yim N (2018) Wnt signaling and bone regeneration: Can't have one without the other. Biomaterials. 196:46-50

21. Olsen JJ, Pohl SÖ-G, Deshmukh A, Visweswaran M, Ward NC, Arfuso F, Agostino M, Dharmarajan A (2017) The role of Wnt signalling in angiogenesis. Clin Biochem Rev 38:131-142

22. Colnot C, Romero DM, Huang S, Helms JA (2005) Mechanisms of action of demineralized bone matrix in the repair of cortical bone defects. Clin Orthop Relat Res:69-78 
23. Taylor SC, Berkelman T, Yadav G, Hammond M (2013) A defined methodology for reliable quantification of Western blot data. Mol Biotechnol 55:217-226

24. Behr B, Leucht P, Longaker MT, Quarto N (2010) Fgf-9 is required for angiogenesis and osteogenesis in long bone repair. PNAS 107: 11853-11858

25. Sawyer A, Lott P, Titrud J, McDonald J (2003) Quantification of tartrate resistant acid phosphatase distribution in mouse tibiae using image analysis. Biotechnic Histochem 78:271-278

26. Schmidt-Rohlfing B, Lemmen SW, Pfeifer R, Pape HC (2012) Osteomyelitis in adults. Diagnostic principles and therapeutic strategies. Unfallchirurg 115:55-66

27. Ling Z, Wu L, Shi G, Chen L, Dong Q (2017) Increased Runx2 expression associated with enhanced Wnt signaling in PDLLA internal fixation for fracture treatment. Exp Ther Med 13:2085-2093

28. Chen Y, Whetstone HC, Lin AC, Nadesan P, Wei Q, Poon R, Alman BA (2007) Beta-catenin signaling plays a disparate role in different phases of fracture repair: implications for therapy to improve bone healing. PLoS Med 4:e249

29. Gaur T, Lengner CJ, Hovhannisyan H, Bhat RA, Bodine PVN, Komm BS, Javed A, van Wijnen AJ, Stein JL, Stein GS, Lian JB (2005) Canonical WNT signaling promotes osteogenesis by directly stimulating Runx2 gene expression. J Biol Chem 280:3313233140

30. Komori T (2006) Regulation of osteoblast differentiation by transcription factors. J Cell Biochem 99:1233-1239

31. Lee M-H, Kwon T-G, Park H-S, Wozney JM, Ryoo H-M (2003) BMP-2-induced Osterix expression is mediated by Dlx 5 but is independent of Runx2. Biochem Biophys Res Commun 309:689694

32. Lee M-H, Kim Y-J, Yoon W-J, Kim J-I, Kim B-G, Hwang Y-S, Wozney JM, Chi X-Z, Bae S-C, Choi K-Y, Cho JY, Choi JY, Ryoo HM (2005) Dlx 5 specifically regulates Runx 2 type II expression by binding to homeodomain-response elements in the Runx2 distal promoter. J Biol Chem 280:35579-35587

33. Ryoo H-M, Lee M-H, Kim Y-J (2006) Critical molecular switches involved in BMP-2-induced osteogenic differentiation of mesenchymal cells. Gene 366:51-57

34. Cho Y-D, Kim W-J, Yoon W-J, Woo K-M, Baek J-H, Lee G, Kim G-S, Ryoo H-M (2012) Wnt3a stimulates Mepe, matrix extracellular phosphoglycoprotein, expression directly by the activation of the canonical Wnt signaling pathway and indirectly through the stimulation of autocrine bmp-2 expression. J Cell Physiol 227: 2287-2296

35. Tanaka S, Tanaka Y, Ishiguro N, Yamanaka H, Takeuchi T (2018) RANKL: a therapeutic target for bone destruction in rheumatoid arthritis. Mod Rheumatol 28:9-16

36. Chiu YG, Ritchlin CT (2017) Denosumab: targeting the RANKL pathway to treat rheumatoid arthritis. Expert Opin Biol Ther 17: $119-128$

37. Wang D, Weng Y, Guo S, Zhang Y, Zhou T, Zhang M, Wang L, Ma J (2018) Platelet-rich plasma inhibits RANKL-induced osteoclast differentiation through activation of Wnt pathway during bone remodeling. Int J Mol Med 41:729-738

38. Amirhosseini M, Madsen RV, Escott KJ, Bostrom MP, Ross FP, Fahlgren A (2018) GSK-3beta inhibition suppresses instabilityinduced osteolysis by a dual action on osteoblast and osteoclast differentiation. J Cell Physiol 233:2398-2408

39. Weivoda MM, Ruan M, Hachfeld CM, Pederson L, Howe A, Davey RA, Zajac JD, Kobayashi Y, Williams BO, Westendorf JJ,
Khosla S, Oursler MJ (2016) Wnt signaling inhibits osteoclast differentiation by activating canonical and noncanonical cAMP/PKA pathways. J Bone Mineral Res 31:65-75

40. Walsh NC, Reinwald S, Manning CA, Condon KW, Iwata K, Burr DB, Gravallese EM (2009) Osteoblast function is compromised at sites of focal bone erosion in inflammatory arthritis. J Bone Miner Res 24:1572-1585

41. Jin H, Wang B, Li J, Xie W, Mao Q, Li S, Dong F, Sun Y, Ke H-Z, Babij P, Tong P, Chen D (2015) Anti-DKK1 antibody promotes bone fracture healing through activation of beta-catenin signaling. Bone 71:63-75

42. Duan Y, Liao AP, Kuppireddi S, Ye Z, Ciancio MJ, Sun J (2007) Beta-catenin activity negatively regulates bacteria-induced inflammation. Lab Investigation 87:613-624

43. Sun J, Hobert ME, Duan Y, Rao AS, He T-C, Chang EB, Madara JL (2005) Crosstalk between NF-kappaB and beta-catenin pathways in bacterial-colonized intestinal epithelial cells. Am J Physiol Gastrointest Liver Physiol 289:G129-G137

44. Niida A, Hiroko T, Kasai M, Furukawa Y, Nakamura Y, Suzuki Y, Sugano S, Akiyama T (2004) DKK1, a negative regulator of Wnt signaling, is a target of the beta-catenin/TCF pathway. Oncogene 23:8520-8526

45. Ahn Y, Sanderson BW, Klein OD, Krumlauf R (2010) Inhibition of Wnt signaling by wise (Sostdc1) and negative feedback from Shh controls tooth number and patterning. Development (Cambridge, England) 137:3221-3231

46. Aurrekoetxea M, Irastorza I, Garcia-Gallastegui P, Jimenez-Rojo L, Nakamura T, Yamada Y, Ibarretxe G, Unda FJ (2016) Wnt/betacatenin regulates the activity of epiprofin/Sp6, SHH, FGF, and BMP to coordinate the stages of odontogenesis. Front Cell Development Biol 4:25

47. Stolina M, Dwyer D, Niu Q-T, Villasenor KS, Kurimoto P, Grisanti M, Han C-Y, Liu M, Li X, Ominsky MS, Ke HZ, Kostenuik PJ (2014) Temporal changes in systemic and local expression of bone turnover markers during six months of sclerostin antibody administration to ovariectomized rats. Bone 67:305-313

48. Florio M, Gunasekaran K, Stolina M, Li X, Liu L, Tipton B, SalimiMoosavi H, Asuncion FJ, Li C, Sun B, Tan HL, Zhang L, Han CY, Case R, Duguay AN, Grisanti M, Stevens J, Pretorius JK, Pacheco E, Jones H, Chen Q, Soriano BD, Wen J, Heron B, Jacobsen FW, Brisan E, Richards WG, Ke HZ, Ominsky MS (2016) A bispecific antibody targeting sclerostin and DKK-1 promotes bone mass accrual and fracture repair. Nat Commun 7:11505

49. Wang N, Xue P, Wu X, Ma J, Wang Y, Li Y (2018) Role of sclerostin and dkk1 in bone remodeling in type 2 diabetic patients. Endocr Res 43:29-38

50. Esteve P, Bovolenta $\mathrm{P}(2010)$ The advantages and disadvantages of Sfrp1 and Sfrp2 expression in pathological events. Tohoku J Exp Med 221:11-17

51. Ding M, Wang X (2017) Antagonism between hedgehog and Wnt signaling pathways regulates tumorigenicity. Oncol Lett 14:63276333

52. McClung MR, Grauer A, Boonen S, Bolognese MA, Brown JP, Diez-Perez A, Langdahl BL, Reginster J-Y, Zanchetta JR, Wasserman SM et al (2014) Romosozumab in postmenopausal women with low bone mineral density. N Engl J Med 370:412-420

Publisher's note Springer Nature remains neutral with regard to jurisdictional claims in published maps and institutional affiliations. 